\title{
Electronic electrical conductivity in n-type silicon
}

\author{
Abebaw Abun Amanu \\ Haramaya University, college of natural and computational science, \\ Department of physics, P. O. Box 138 Dire Dawa, Ethiopia
}

E-mail: meseret.abun@gmail.com

(Received 30 September 2014, accepted 26 May 2015).

\begin{abstract}
The electrical conductivity of n-type silicon depends on the doping concentration which varies from $10^{22}-10^{26} / \mathrm{m}^{3}$ at a given temperature $300^{\circ} \mathrm{K}$ where ionized impurity scattering is the dominant scattering mechanism. This work founds that the electrical conductivity of n-type silicon increases as the electron concentration increases as the result of doping. When the electron concentration increases, the Fermi energy increases from the result of the Fermi level increment.
\end{abstract}

Keywords: Doping concentration, Fermi energy, Electrical conductivity.

\section{Resumen}

La conductividad eléctrica del silicio de tipo $\mathrm{n}$ depende de la concentración de dopaje, la cual varía de $10^{22}-10^{26} / \mathrm{m}^{3}$ a una temperatura de $300^{\circ} \mathrm{K}$, donde la dispersión de impurezas ionizadas es el mecanismo de dispersión dominante. Este trabajo demuestra que la conductividad eléctrica del silicio de tipo n, aumenta conforme la concentración de electrones aumenta como resultado de dopaje. Cuando aumenta la concentración de electrones, la energía Fermi aumenta como resultado del incremento del nivel Fermi.

Palabras clave: Concentración de dopaje, Energía de Fermi, Conductividad eléctrica.

PACS: 01.40.-d, 03.75.Lm,

ISSN 1870-

9095

\section{INTRODUCTION}

Semiconductors are materials at the heart of many electronic devices, such as transistors, switches, diodes, photovoltaic cells, etc. Silicon is widely used now a day with several applications in light emitting diodes, semiconductor lasers, microwaves lasers, and others specialized areas [1].

Semiconductor is a material that has a conductance value between that of an insulators and conductors. In addition, their resistance between them. They are only different from insulators because of conduction brought about by thermally generated charge carries (extrinsic conduction) called dopants in semiconductor devices only extrinsic conduction is desirable, the charge carries are electrons and holes [1].

By adding the right kind of dopants it is possible to make semiconductor materials, n-type materials and p-type materials. If such impurities contribute a significant fraction of the conduction band electrons and /or valance band holes, one speaks of an "extrinsic semiconductors" [3].

The objective of this research is:

To show the relationship between Fermi energy the electron concentration

To show the relationship between the electrical conductivity and the electron concentration
To derive the mathematical expression for electrical conductivity and to calculate the numerical values in n-type silicon for different doping concentrations in the range $10^{16} / \mathrm{cm}^{3}-10^{18} / \mathrm{cm}^{3}$.

The physical significance of this research is to understand the electrical conductivity of the n-type silicon that has so many applications in the electronic world.

\section{CONSTANT ENERGY SURFACES OF CONDUCTION ENERGY BAND STRUCTURE AND THE QUANTUM DENSITY OF STATES OF N-TYPE SILICON}

The system under consideration is n-type silicon. There are six equivalent constant energy ellipsoids for electron in silicon. These are six equivalents energy minimum along the six $\{100\}$ directions [3]. The constant energy surfaces as seen by the $\{100\}$ plane through the center of the first Brillion zone in p-space with axis of symmetry in the $\mathrm{x}$-axis will have energy given by an expression of the form:

$$
E=E_{c}+\frac{P_{1}^{2}}{2 m_{1}^{*}}+\frac{P_{2}^{2}}{2 m_{2}^{*}}+\frac{P_{3}^{2}}{2 m_{3}^{*}} .
$$




\section{Abebaw Abun Amanu}

Where $\mathrm{m}_{1}{ }^{*}=\mathrm{m}_{1}=0.92 \mathrm{~m}_{0}$ is the longitudinal effective mass and $\mathrm{m}_{2}{ }^{*}=\mathrm{m}_{3}{ }^{*}=\mathrm{m}_{\mathrm{T}}=0.91 \mathrm{~m}_{0}$ is the transverse effective mass.

By adding appropriate transformation to anew $\mathrm{P}$ ' coordinate system in which the constant energy surface because spherical. The energy can be expressed in the form:

$$
E=E_{c}+\frac{P^{\prime 2}}{2 m_{n}^{*}}
$$

Where $m_{n}^{*}=\left(m_{1}^{*} m_{2}^{* 2}\right)^{\frac{1}{2}} M_{v}^{\frac{2}{3}}$ is the density of the states effective mass and $M_{v}=6$ number of equivalent energy valleys. The number of quantum states in P-space in the energy range $\mathrm{E}+\mathrm{dE}$ is:

$$
\rho(E)=\frac{8 \sqrt{2}}{h^{3}} \pi m_{n}^{* \frac{3}{2}}\left(E-E_{c}\right)^{\frac{1}{2}}
$$

If we measure energy from the bottom of conduction $\mathrm{Ec}=0$, then $\rho(E)$ can be expressed as:

$$
\rho(E)=\frac{8 \sqrt{2}}{h^{3}} \pi m_{n}^{* \frac{3}{2}}(E)^{\frac{1}{2}} .
$$

\section{FERMI DIRAC STATISTICS FOR N-TYPE SILICON}

The number of states per unit volume between $E$ and $E+d E$ in allowed band $\rho(E) d E$ can be calculated from the volume between $E$ and $d E$ in an allowed band, $\rho(E) d E$ can be calculated from the volume between $E$ and $d E$ in kspace divided by the volume of a single state in $\mathrm{k}$-space. If the shape of the energy surface in the k-space is known for a given material, therefore, $\rho(E) d E$ can be calculated. If $f(E)$ is the probability that a state with energy $E$ will be occupied states is given by an expression of the form:

$$
n=\int_{0}^{\infty} f(E) \rho(E) d E
$$

Where $n$ is the number of electrons in the conduction band, now the function $(E)$, the profanity that a state with energy $E$ will be occupied, is just the Fermi distribution function.

For electron occupation of the conduction band, $f(E)$ can be expressed as:

$$
f(E)=\frac{1}{\exp \frac{E-E_{f}}{k_{B} T}+1} .
$$

Where $E_{f}$ is the Fermi energy:

To derive the number of electrons in the conduction band, use the above equations. Substitute Eq. (4) and Eq. (6) into Eq. (5), i.e.

$$
n=\int_{0}^{\infty}\left(\frac{1}{\exp \left(\frac{E-E_{f}}{K_{B} T}\right)+1}\right)\left(\frac{8 \sqrt{2}}{h^{3}} \pi m_{n}^{* \frac{3}{2}}(E)^{\frac{1}{2}}\right) d E
$$

$$
n=\left(\frac{8 \sqrt{2}}{h^{3}} \pi m_{n}^{* \frac{3}{2}}\right) \int_{0}^{\infty}\left(\frac{(E)^{\frac{1}{2}}}{\exp \left(\frac{E-E_{f}}{K_{B} T}\right)+1}\right) d E .
$$

In addition, the normalized electron concentration $n_{n}$ is:

$$
n_{n}=\frac{n}{10^{25} / m^{3}}
$$

We assume that the total mobile electron concentration in the conduction band is equal to donor concentration $\mathrm{N}_{\mathrm{d}}$ that varies from $10^{22}-10^{26} / \mathrm{m}^{3}$ in our calculation.

\section{BOLTZMANN TRANSPORT EQUATIONS}

The conductivity of a substance is determined by the concentration and mobility of charge carriers. The probability of electrons occupying a unit volume of phase space with the center at point $(\mathrm{x}, \mathrm{k})$ at the moment of time $\mathrm{t}$ is $f(x, k, t)[5]$. That is to say $f(x, k, t)$ is the distribution function for no equilibrium state the distribution function will change with time, the nature of change being dependent on which process predominates; the change due to the action of the electric field (F), and as a result of charge carrier collision(C).

$$
\frac{d f}{d t}=\left(\frac{\partial f}{\partial t}\right)_{F}+\left(\frac{\partial f}{\partial t}\right)_{C} .
$$

Where:

$$
\frac{d f}{d t}=\left(\frac{\partial f}{\partial t}\right)_{C}+\frac{\partial f}{\partial x} \frac{d x}{d t}+\frac{\partial f}{\partial k} \frac{d k}{d t}
$$

Or

$$
\begin{gathered}
\frac{d f}{d t}=\left(\frac{\partial f}{\partial t}\right)_{C}+\frac{\partial f}{\partial x} \frac{d x}{d t}+\frac{\partial f}{\partial v} \frac{d v}{d t} \\
=\left(\frac{\partial f}{\partial t}\right)_{C}+v_{x} \frac{\partial f}{\partial x}+a_{x} \frac{\partial f}{\partial v}
\end{gathered}
$$

For the present, we want to avoid excessive complications by means of relaxation time approximations for $\left(\frac{\partial f}{\partial t}\right)_{C}$. The effect of collisions is always to restore a local equilibrium situation described by the distribution function $f_{0}(r, v, t)$. Let us further assume that if the electron distribution is distributed from the local equilibrium value $f_{0}$, then the effect of the collision is simply to restore $f$ to the local equilibrium value $f_{0}$ exponentially with a relaxation time $\tau$ which is the order of the time between electron collisions with ion, i.e.:

$$
\left(\frac{\partial f}{\partial t}\right)_{C}+v_{x} \frac{\partial f}{\partial x}+a_{x} \frac{\partial f}{\partial v_{x}}
$$

From the relations:

$$
F_{x}=m_{n}^{*} a_{x}
$$


Substitute Eq. (3.6) into Eq. (3.5):

$$
\frac{d f}{d t}=\left(\frac{\partial f}{\partial t}\right)_{C}+v_{x} \frac{\partial f}{\partial x}+\frac{f_{x}}{m_{n}^{*}} \frac{\partial f}{\partial v_{x}} .
$$

Where $m_{n}^{*}$ is the effective mass of an electron.

From the general relation of the electrical force and the electric field, we get the below equation.

Where $e=1.6 \times 10^{-19} \mathrm{C}$, electric charge and $E_{x}$ is the electric field in the $\mathrm{x}$-direction.

$$
\frac{d f}{d t}=\left(\frac{\partial f}{\partial t}\right)_{C}+v_{x} \frac{\partial f}{\partial x}-\frac{e E_{x}}{m_{n}^{*}} \frac{\partial f}{\partial v_{x}} .
$$

For the steady state condition, the electron distribution is independent of time, i.e. $\frac{d f}{d t}=0$, Eq. (17) becomes:

$$
v_{x} \frac{\partial f}{\partial x}-\frac{e E_{x}}{m_{n}^{*}} \frac{\partial f}{\partial v_{x}}=-\left(\frac{\partial f}{\partial t}\right)_{C} .
$$

Where in the relaxation time approximation:

$$
\left(\frac{\partial f}{\partial t}\right)_{C}=\frac{f-f_{0}}{\tau} .
$$

And

$$
v_{x} \frac{\partial f}{\partial x}-\frac{e E_{x}}{m_{n}^{*}} \frac{\partial f}{\partial v_{x}}=-\left(\frac{\partial f}{\partial t}\right)_{C}=-\frac{f-f_{0}}{\tau} .
$$

\section{ELECTRON SCATTERING MECHANISM}

There are different scattering mechanisms like acoustic phonon scattering, ionized impurity scattering, carriercarrier scattering among others responsible for the resistivity of the material. Conwell and Weisskopf have calculated the rate of change of distribution function due to ionized impurity scattering by using the following assumptions:

i. In the electron ionized impurity scattering only the direction of electrons changes

ii. An electron gets scattered by a single ion at a time $i$. $e$. by the one which is closest to it at that particular instant of time.

Therefore one can express the number of electrons per unit volume per second into a solid angle $d \Omega^{\prime}$ at $\theta^{\prime}, \Phi^{\prime}$ as:

$$
N f(v, \theta, \Phi) \sigma\left(\theta, \theta^{\prime}\right) v d \Omega .
$$

Where $N$ is the number of electron per unit volume.

$N f(v, \theta, \Phi) d \Omega$ is the number of electrons per unit volume with solid angle $d \Omega$.

$$
\sigma\left(\theta, \theta^{\prime}\right)=\left(\frac{z e^{2}}{8 \pi \varepsilon_{0} \varepsilon_{d}}\right) \frac{1}{\sin ^{4}\left(\theta-\theta^{\prime}\right) / 2} .
$$

Electronic electrical conductivity in n-type silicon Is the Rutherford scattering cross-section and $\mathrm{v}$ is the relative velocity between electron and ion and can be taken as electron velocity.

The Conwell and Weisskopf formula for ionized impurity relaxation time is:

$$
\tau=\tau_{0}\left(\frac{E}{K_{B} T}\right)^{\frac{3}{2}}=\tau_{0} \varepsilon^{\frac{3}{2}} .
$$

Where $\varepsilon$ is the dimensionless kinetic energy. Among varies scattering mechanisms responsible for resistivity in the temperature range $77-300^{\circ} \mathrm{K}$ and for electron concentration, $n \geq 10^{24} / \mathrm{m}^{3}$ the ionized impurity scattering is the dominant scattering mechanism. We shall use the above expression of relaxation time for ionized impurity scattering in subsequent sections to obtain the explicit expression for thermal conductivity.

\section{ELECTRICAL CONDUCTIVITY}

Electrical conduction is transport processes resulting from the motion of charge carriers under the action of internal or external field. Conductivity of n-type silicon in which the conductivity is due to the excess electrons. Current is defined as the time rate at which charge is transported across a given surface in a direction normal to it, the current will depend on both number of charges free to move and the speeds at which they move. Electrical conduction takes place as a result of the motion of the free electrons under the action of an applied electric field [6].

\section{DERIVATION OF ELECTRICAL CONDUCTIVITY}

Current is defined as the time rate at which charge is transported across a given surface in a direction normal to it, the current will depend on both the number of charges free to move and the speeds at which they move.

The electrical current density is given by:

$$
\begin{gathered}
J_{x}=-\frac{2 e m_{n}^{* 3}}{h^{3}} \iiint v_{x} f d v_{y} d v_{z} . \\
J_{x}=-\frac{2 e m_{n}^{* 3}}{h^{3}} \iiint v_{x} f d^{3} v .
\end{gathered}
$$

Where $f$ can be expanded as $f=f_{0}+v_{x} f_{x}$ to the first order approximation for weak/normal dc electric field.

$$
J_{x}=-\frac{2 e m_{n}^{* 3}}{h^{3}} \iiint v_{x}\left(f_{0}+v_{x} f_{x}\right) d^{3} v .
$$

$f_{0}+v_{x} f_{x} \approx v_{x} f_{x}$, since no current flows in equilibrium, $f_{0}$ does not contribute to the electric field current. Thus:

$$
J_{x}=-\frac{2 e m_{n}^{* 3}}{h^{3}} \iiint v_{x}^{2} f_{x} d^{3} v
$$


Abebaw Abun Amanu

The Boltzmann transport equation in the presence of a d. c electric field $E_{x}$ in the x direction is calculated by:

$$
\frac{e E_{x}}{m_{n}^{*}} \frac{\partial}{\partial v_{x}}\left(f_{0}+v_{x} f_{x}\right)=-\frac{f-f_{0}}{\tau}=-\frac{f_{x} v_{x}}{\tau}
$$

$\frac{\partial f}{\partial v_{x}} \approx \frac{\partial f_{0}}{\partial v_{x}}$, leaving the higher order terms in the expansion of $f$. From the above Eq. (28) relations:

$$
\frac{e E_{x}}{m_{n}^{*}} \frac{\partial f_{0}}{\partial v_{x}}=-\frac{f_{x} v_{x}}{\tau} .
$$

Then:

$$
f_{x}=\frac{e E_{x}}{m_{n}^{*} v_{x}} \frac{\tau \partial f_{0}}{\partial v_{x}}
$$

Thus:

$$
J_{x}=-\frac{2 e m_{n}^{* 3}}{h^{3}} \iiint v_{x}^{2} f_{x} d^{3} v
$$

By using solid angle relations:

$$
d^{3} v=d v_{x} d v_{y} d v_{z}=v^{2} \sin \theta d \theta d \Phi d v
$$

Substitute this Eq. (32) into Eq. (30):

$$
\begin{gathered}
J_{x}=-\frac{2 e m_{n}^{* 3}}{h^{3}} \int_{0}^{2 \pi} \int_{0}^{\pi} \int_{0}^{\infty} v_{x}^{2} f_{x}\left(v^{2} \sin \theta d \theta d \Phi d v\right) \\
=-\frac{2 e m_{n}^{* 3}}{h^{3}} \int_{0}^{2 \pi} d \Phi \int_{0}^{\pi} \int_{0}^{\infty} v_{x}^{2} f_{x}\left(v^{2} \sin \theta d \theta d v\right) \\
=-\frac{4 e m_{n}^{* 3}}{h^{3}} \int_{0}^{\pi} \int_{0}^{\infty} v_{x}^{2} f_{x}\left(v^{2} \sin \theta d \theta d v\right) .
\end{gathered}
$$

From the vector $\mathrm{v}$ and angle $\theta$ relations:

$$
v_{x}=v \cos \theta
$$

Substitute Eq. (35) into Eq. (34):

$$
\begin{aligned}
J_{x} & =-\frac{4 e m_{n}^{* 3}}{h^{3}} \int_{0}^{\pi} \int_{0}^{\infty}(v \cos \theta)^{2} f_{x}\left(v^{2} \sin \theta d \theta d v\right) \\
& =-\frac{4 e m_{n}^{* 3}}{h^{3}} \int_{0}^{\pi} \int_{0}^{\infty}(v)^{4} f_{x} \cos ^{2} \theta \sin \theta d \theta d v
\end{aligned}
$$

By using the relations of the above equations, we can drive the below equation.

$$
\begin{aligned}
J_{x} & =-\frac{4 e m_{n}^{* 3}}{h^{3}} \int_{0}^{\pi} \int_{0}^{\infty} v^{4}\left(\frac{e E_{x} \tau}{m_{n}^{*}} \frac{\partial f_{0}}{\partial E}\right) \cos ^{2} \theta \sin \theta d \theta d v \\
& =-\frac{4 e m_{n}^{* 3} E_{x}}{h^{3}} \int_{0}^{\pi} \int_{0}^{\infty} v^{4}\left(\frac{e \tau}{m_{n}^{*}} \frac{\partial f_{0}}{\partial E}\right) \cos ^{2} \theta \sin \theta d \theta d v \\
J_{x} & =-\frac{4 e^{2} m_{n}^{* 3} E_{x}}{h^{3}} \int_{0}^{\pi} \cos ^{2} \theta \sin \theta d \theta \int_{0}^{\infty} v^{4} \tau \frac{\partial f_{0}}{\partial E} d v
\end{aligned}
$$

$$
\int_{0}^{\infty} \cos ^{2} \theta \sin \theta d \theta
$$

Using integration by substitution, we can integrate the above equation, i.e. let $\cos \theta=u$, then $-\sin d \theta=d u$.

Replacing the first thing in $u$, then:

$$
\begin{gathered}
\text { i.e. }\left[-\frac{\cos ^{3} \theta}{3}\right]_{0}^{\pi} \\
=-\frac{1}{3}\left(\cos ^{3} \pi-\cos ^{3} 0\right)=\frac{2}{3} .
\end{gathered}
$$

Then:

$$
J_{x}=-\frac{8 e^{2} m_{n}^{* 3} E_{x}}{3 h^{3}} \int_{0}^{\infty} v^{4} \tau \frac{\partial f_{0}}{\partial E} d v
$$

Substitute Eq. (23) into Eq. (39), then:

$$
J_{x}=-\frac{8 e^{2} m_{n}^{* 3} E_{x}}{3 h^{3}} \int_{0}^{\infty} v^{4} \tau \varepsilon^{\frac{3}{2}} \frac{\partial f_{0}}{\partial E} d v
$$

From the relation of $\mathrm{v}$ and energy $\mathrm{E}$ :

$$
d\left(\frac{1}{2} m_{n}^{*} v^{2}\right)=d E
$$

$$
v m_{n}^{*} d v=d E, v d v=\frac{d E}{m_{n}^{*}} .
$$

Substitute Eq. (42) into Eq. (40):

$$
\begin{aligned}
& J_{x}=-\frac{8 \pi e^{2} m_{n}^{* 3} E_{x}}{3 h^{3}} \int_{0}^{\infty} v^{3}\left(\tau_{0} \varepsilon^{\frac{3}{2}}\right) \frac{\partial f_{0}}{\partial E}\left(\frac{d E}{m_{n}^{*}}\right) . \\
& \frac{1}{2} m_{n}^{*} v^{2}=E, v^{2}=\frac{2 E}{m_{n}^{*}}, \text { then, } v=\sqrt{\frac{2 E}{m_{n}^{*}}} .
\end{aligned}
$$

Substitute Eq. (44) into Eq. (43):

$$
\begin{aligned}
J_{x} & =-\frac{8 \pi e^{2} m_{n}^{* 3} E_{x}}{3 h^{3}} \int_{0}^{\infty}\left(\frac{2 E}{m_{n}^{*}}\right)^{\frac{3}{2}}\left(\tau_{0} \varepsilon^{\frac{3}{2}}\right) \frac{\partial f_{0}}{\partial E} d E \\
& =-\frac{16 \sqrt{2} \pi e^{2} m_{n}^{* 3} E_{\chi} \tau_{0}}{3 h^{3} m_{n}^{* \frac{5}{2}}} \int_{0}^{\infty} E^{\frac{3}{2}} \varepsilon^{\frac{3}{2}} \frac{\partial f_{0}}{\partial E} d E
\end{aligned}
$$

Change all the energies that are the equation becomes dimensionless kinetic energy of an electron.

$$
\varepsilon=\frac{E}{K_{B} T}, E=\varepsilon K_{B} T .
$$

Substitute Eq. (47) into Eq. (46):

$$
\begin{gathered}
J_{x}=-\frac{16 \sqrt{2} \pi e^{2} m_{n}^{* \frac{1}{2}} E_{x} \tau_{0}}{3 h^{3}} \int_{0}^{\infty}\left(\varepsilon K_{B} T\right)^{\frac{3}{2}} \varepsilon^{\frac{3}{2}} \frac{\partial f_{0}}{\partial\left(\varepsilon K_{B} T\right)} d\left(\varepsilon K_{B} T\right) \\
J_{x}=-\frac{16 \sqrt{2} \pi e^{2} m_{n}^{* \frac{1}{2}} E_{x} \tau_{0}}{3 h^{3}} \int_{0}^{\infty}\left(\varepsilon K_{B} T\right)^{\frac{3}{2}} \varepsilon^{\frac{3}{2}} \frac{\partial f_{0}}{\partial \varepsilon} d \varepsilon
\end{gathered}
$$




$$
J_{x}=\frac{16 \sqrt{2} \pi e^{2} m_{n}^{* \frac{1}{2}} E_{x} \tau_{0}}{3 h^{3}} \int_{0}^{\infty}\left(\varepsilon K_{B} T\right)^{\frac{3}{2}} \varepsilon^{\frac{3}{2}}\left(-\frac{\partial f_{0}}{\partial \varepsilon} d \varepsilon\right) .
$$

By using integration by parts we can solve the above complex mathematical equation. So:

$$
\begin{gathered}
\int_{0}^{\infty} \varepsilon^{3}\left(-\frac{\partial f_{0}}{\partial \varepsilon} d \varepsilon\right) \\
\int d v=\int-\frac{\partial f_{0}}{\partial \varepsilon} d \varepsilon, v=f_{0}, \\
\text { and }, u=\varepsilon^{3}, \text { and } d u=3 \varepsilon^{2} d \varepsilon \\
u v-\int v d u=\left[-\varepsilon^{3}\right]_{0}^{\infty}+3 \int_{0}^{\infty} f_{0} \varepsilon^{2} d \varepsilon \\
=0+3 \int_{0}^{\infty} f_{0} \varepsilon^{2} d \varepsilon .
\end{gathered}
$$

By substituting:

Finally:

$$
\begin{aligned}
f_{0} & =\frac{1}{1+\exp \left(\varepsilon+\varepsilon_{f}\right)} \\
& =3 \int \frac{\varepsilon^{2} d \varepsilon}{1+\exp \left(\varepsilon-\varepsilon_{f}\right)} .
\end{aligned}
$$

$$
\begin{aligned}
& J_{x}=-\frac{16 \sqrt{2} \pi e^{2} m_{n}^{* \frac{1}{2}} E_{x} \tau^{3}\left(K_{B} T\right)^{\frac{3}{2}}}{3 h^{3}} 3 \int_{0}^{\infty} \frac{\varepsilon^{2} d \varepsilon}{1+\exp \left(\varepsilon-\varepsilon_{f}\right)} \\
& J_{x}=-\frac{16 \sqrt{2} \pi e^{2} m_{n}^{* \frac{1}{2}} E_{x} \tau^{3}\left(K_{B} T\right)^{\frac{3}{2}}}{h^{3}} \int_{0}^{\infty} \frac{\varepsilon^{2} d \varepsilon}{1+\exp \left(\varepsilon-\varepsilon_{f}\right)}
\end{aligned}
$$

From the general relation of $J_{x}=\sigma_{x} E_{x}$

$$
\begin{gathered}
\sigma_{x}=\frac{J_{x}}{E_{x}} \\
\sigma_{x}=\frac{16 \sqrt{2} \pi e^{2} m_{n}^{* \frac{1}{2}} \tau_{0}{ }^{3}\left(K_{B} T\right)^{\frac{3}{2}}}{h^{3}} \int_{0}^{\infty} \frac{\varepsilon^{2} d \varepsilon}{1+\exp \left(\varepsilon-\varepsilon_{f}\right)} \\
\sigma_{x}=\frac{8 \sqrt{2} \pi e^{2} m_{n}^{* \frac{3}{2}} \tau_{0}^{3}\left(K_{B} T\right)^{\frac{3}{2}}\left(2 e^{2} \tau_{0}\right)}{h^{3} m_{n}^{*}} \int_{0}^{\infty} \frac{\varepsilon^{2} d \varepsilon}{1+\exp \left(\varepsilon-\varepsilon_{f}\right)}
\end{gathered}
$$

Substitute Eq. (8) into Eq. (58), i.e.:

$$
\begin{gathered}
\sigma_{x}=\frac{2 n e^{2} \tau_{0}}{\int_{0}^{\infty} \frac{(\varepsilon)^{\frac{1}{2}}}{1+\exp \left(\varepsilon-\varepsilon_{f}\right)}}\left(m_{n}^{* \frac{1}{2}}\right) \int_{0}^{\infty} \frac{\varepsilon^{2} d \varepsilon}{1+\exp \left(\varepsilon-\varepsilon_{f}\right)} . \\
\sigma_{x}=\frac{2 n e^{2} \tau_{0}}{m_{n}^{*}} \frac{\int_{0}^{\infty} \frac{\varepsilon^{2} d \varepsilon}{1+\exp \left(\varepsilon-\varepsilon_{f}\right)}}{\int_{0}^{\infty} \frac{(\varepsilon)^{\frac{1}{2}}}{1+\exp \left(\varepsilon-\varepsilon_{f}\right)}}
\end{gathered}
$$

Electronic electrical conductivity in n-type silicon

$$
\sigma_{x}^{d}=\frac{\sigma_{x}}{\frac{n e^{2} \tau_{0}}{m_{n}^{*}}}=\frac{2 F_{2}\left(\varepsilon_{f}\right)}{F_{\frac{1}{2}}\left(\varepsilon_{f}\right)}
$$

This Eq. (61) is known as the normalized electrical conductivity.

\section{NUMERICAL CALCULATION OF ELECTRICAL CONDUCTIVITY}

To calculate numerical values of the normalized Fermi energy $\varepsilon_{f}$ and the dimensionless electrical conductivity $\sigma_{x}^{d}$ for the given electron concentration, use the formula for electron concentration.

$$
n=\left(\frac{8 \pi m_{n}^{* \frac{3}{2}} \sqrt{2}}{h^{3}}\right) \int_{0}^{\infty}\left(\left(K_{B} T\right)^{\frac{1}{2}}\left(\frac{E}{K_{B} T}\right)^{\frac{1}{2}}\left(K_{B} T\right) \frac{d\left(\frac{E}{K_{B} T}\right)}{\exp \left(\frac{E-E_{f}}{K_{B} T}\right)+1}\right) .
$$

Where

Whit

$$
T=300^{0} K, m_{n}^{*}=1.18 m_{0}
$$

$m_{0}=9.11 \times 10^{-31} \mathrm{~kg}$, and $\varepsilon=\frac{E}{K_{B} T}$ is the dimensionless kinetic energy.

$$
\begin{aligned}
& n=\left(\frac{8 \pi m_{n}^{* \frac{3}{2}} \sqrt{2}}{h^{3}}\right) \int_{0}^{\infty}\left(\left(K_{B} T\right)^{\frac{3}{2}} \varepsilon^{\frac{1}{2}} \frac{d \varepsilon}{1+\exp \left(\varepsilon-\varepsilon_{f}\right)}\right) . \\
& n=\left(\frac{8 \pi m_{n}^{* \frac{3}{2}} \sqrt{2}}{h^{3}}\right)\left(K_{B} T\right)^{\frac{3}{2}} \int_{0}^{\infty}\left(\varepsilon^{\frac{1}{2}} \frac{d \varepsilon}{1+\exp \left(\varepsilon-\varepsilon_{f}\right)}\right) .
\end{aligned}
$$

By substituting the numerical values of the constants, will got:

$$
\begin{gathered}
=3.62 \int_{0}^{\infty}\left(\varepsilon^{\frac{1}{2}} \frac{d \varepsilon}{1+\exp \left(\varepsilon-\varepsilon_{f}\right)}\right), \\
F_{p}\left(\varepsilon_{f}\right)=\int_{0}^{\infty}\left(\varepsilon^{p} \frac{d \varepsilon}{1+\exp \left(\varepsilon-\varepsilon_{f}\right)}\right) .
\end{gathered}
$$

This integral is known as Fermi integral.

To get the dimensionless Fermi energy using the given value of normalized electron concentration those are shown in Table I.

Using Equation (9) for normalized doping concentration $n_{n}$ and the integral Equation (64). The integral Equation (64) for electron concentration is difficult to evaluate because the normalized Fermi energy $\varepsilon_{f}$ is unknown.

By using iteration method in such a way that for a given arbitrary value of $\varepsilon_{f}$ the left side of the integral Equation (64) can be evaluated by using a Mathematica 5.1 software program. The value of the normalized electron concentration obtained by this numerical calculation will be compared with the known initial value $n_{n}=0.04628$. I 
Abebaw Abun Amanu

continue my calculation until I get the precise value of the normalized Fermi energy $\varepsilon_{f}$ corresponding to the given normalized electron concentration $n_{n}=0.04626$.

Therefore, I get the value on the right side of Eq. (64) which must be approximately equals to the value of $n_{n}$ on the left side of Eq. (64) with an error in the order of $10^{-3}$. This iteration method is used again to get the other values of the normalized Fermi energy $\varepsilon_{f}$ corresponding to the given electron concentration $n_{n}$ in the table. These values are used to calculate dimensionless electrical conductivity $\sigma_{x}^{d}$ corresponding to the given value of the normalized electron concentration $n_{n}$ as shown in the below table.

\section{TABLE I.}

\begin{tabular}{|c|c|c|}
\hline $\begin{array}{c}\text { Normalized electron } \\
\text { concentration }\left(n_{n}\right)\end{array}$ & $\begin{array}{l}\text { Dimensionless } \\
\text { Fermi energy }\end{array}$ & $\begin{array}{l}\text { Normalized elec- } \\
\text { trical conductivity }\end{array}$ \\
\hline 0.0462845 & -4.23354 & 4.528399 \\
\hline 0.12039 & -3.26945 & 4.55227 \\
\hline 0.1605 & -2.97748 & 4.565182 \\
\hline 0.240398 & -2.56469 & 4.590962 \\
\hline 0.5095 & -1.80185 & 4.675266 \\
\hline 0.750925 & -1.36966 & 4.756477 \\
\hline 1.0009 & -1.05497 & 4.837995 \\
\hline 1.50085 & -0.59533 & 5.002002 \\
\hline 2.0008 & -0.25354 & 5.167141 \\
\hline 2.50075 & 0.023557 & 5.333409 \\
\hline 3.0007 & 0.259635 & 5.500866 \\
\hline 3.50065 & 0.46734 & 5.669359 \\
\hline 4.0006 & 0.65422 & 5.838826 \\
\hline 4.50055 & 0.825143 & 6.009266 \\
\hline 5.0005 & 0.983438 & 6.180707 \\
\hline 5.50045 & 1.131475 & 6.352981 \\
\hline 6.0004 & 1.27101 & 6.526144 \\
\hline 6.50035 & 1.403375 & 6.700136 \\
\hline 7.0003 & 1.529607 & 6.874981 \\
\hline 7.50025 & 1.65025 & 7.050141 \\
\hline 8.0002 & 1.766795 & 7.226896 \\
\hline 8.50015 & 1.878955 & 7.407064 \\
\hline 9.0001 & 1.987462 & 7.581702 \\
\hline 9.50005 & 2.092687 & 7.760182 \\
\hline 10 & 2.19496 & 7.939213 \\
\hline
\end{tabular}

\section{ANALYSIS AND DISCUSSION ON THE RESULT}

The numerical values are used to draw the graph of normalized Fermi energy $\varepsilon_{f} \quad v s$. normalized electron concentration $n_{n}$.

Again, the numerical values are used to draw the graph of dimensionless electrical conductivity $\left(\sigma_{x}^{d}\right) v s$. normalized electron concentration $n_{n}$.
When we see the first graph, the normalized Fermi energy increases as the doping concentration or the normalized electron concentration $n_{n}$ increases.

When we increase the normalized electron concentration, by doping it from time to time, the Fermi energy level increases with it.

We get negative Fermi energy when the location of the Fermi level is below the bottom of the conduction band and a positive Fermi energy when the location of the Fermi level is above the bottom of the conduction band.

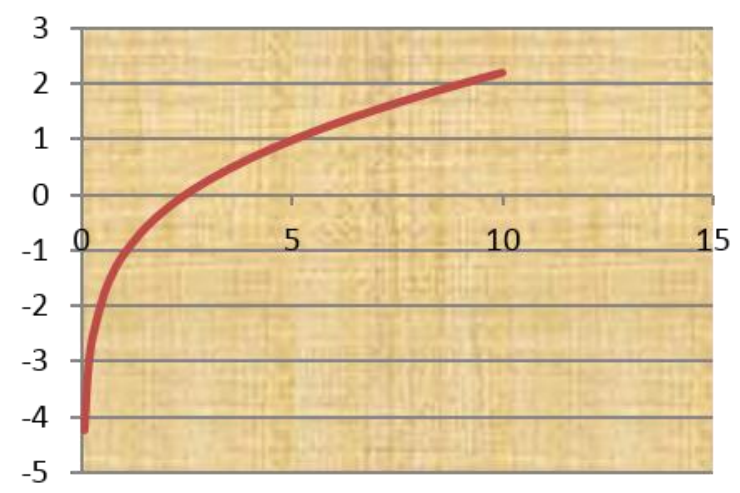

Normalized electron concentration

FIGURE 1. Dimensionless Fermi energy vs. normalized electron concentration.

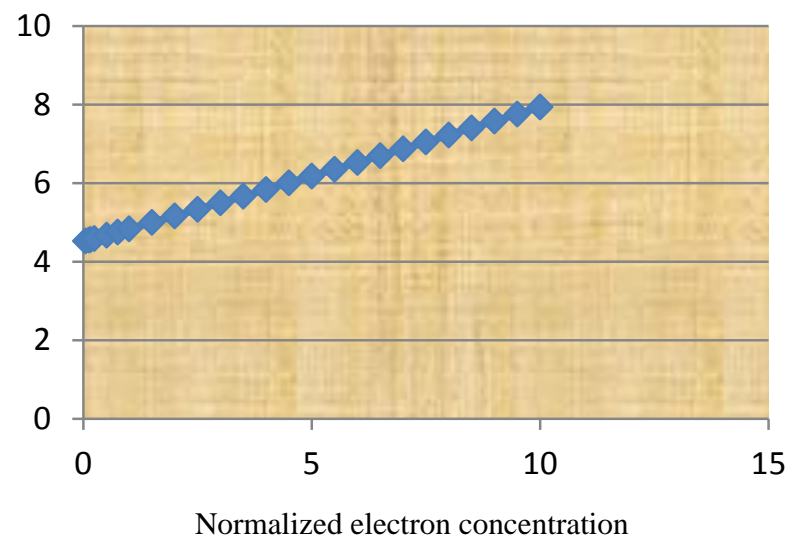

FIGURE 2. Normalized electrical conductivity $v s$. normalized electron concentration.

The graph of normalized electrical conductivity $\left(\sigma_{x}^{d}\right) v s$. normalized electron concentration $n_{n}$ shows that the electrical conductivity of the semiconductor increases by increasing the electron concentration in the conduction band as a result of doping. 


\section{CONCLUSION}

In this research, investigation of how the electrical conductivity of n-type silicon depends on the doping concentration which varies from $10^{22}-10^{26} / \mathrm{m}^{3}$ at a given temperature $300^{\circ} \mathrm{K}$ where ionized impurity scattering is the dominant scattering mechanism.

The paper found that the electrical conductivity of ntype silicon increases as the electron concentration increases, the Fermi energy increases from the result of the Fermi level increases.

\section{REFERENCES}

[1] Warnes, L., Electronic and electrical engineering: Principles and practice, (Palgrave Macmillan, New York, 1995.

[2] Maheshwari, L. K. \& Anand, M. M. S., Laboratory manual for introductory electronic experiments, (New Age International, New Delhi, 2000).

[3] Ascfroft, N. W. \& Mermin, D., Solid state physics, (Brooks Cole, Boston, 1976).

[4] Floyd, T. L. \& Buchla, D. M., Electronics fundamentals: Circuits, devices \& applications, $4^{\text {th }} \mathrm{Ed}$. (Pearson education limited, Harlow, 1998).

[5] Reif, F., Foundamental of statistical and thermal physics, (Mac Graw Hills, Boston, 1985).

[6] Adler, R. B., Smith, A. C. \& Longini, R. L., Introduction to semiconductor physics, (John Wiley \& Sons, New York, 1964). 